\title{
Photoemission of Positronium from Si
}

\author{
D. B. Cassidy, T. H. Hisakado, H. W. K. Tom, and A. P. Mills, Jr. \\ Department of Physics and Astronomy, University of California, Riverside, California 92521-0413, USA
} (Received 9 May 2011; published 12 July 2011)

\begin{abstract}
We have observed that the amount of positronium (Ps) emitted from the surface of $p$-Si(100) is substantially increased if the sample is irradiated with $532 \mathrm{~nm}$ laser light just prior to the implantation of positrons. The energy of the emitted Ps has a constant value of $\sim 0.16 \mathrm{eV}$ and is independent of the $\mathrm{Si}$ temperature and the applied laser fluence, while the photoemission yield depends on both of these parameters. These observations are consistent with Ps production via a previously observed excitonlike positron surface state that is populated in response to the production of electron-hole pairs in the Si. Possible applications of Ps photoemission include probing surface electron dynamics on $\mathrm{Si}$, the generation of ultrashort Ps or positron pulses using ps lasers, and efficient production of Ps in cryogenic environments.
\end{abstract}

The simple act of shining light onto a material and observing the subsequent emission of electrons [1] revealed in a dramatic fashion the quantum nature of light and matter, and played an important role in the early development of quantum physics [2]. More recently, the photoelectric effect has been used with great success to obtain information concerning the electronic structure of materials [3]. Direct electron emission in an atomic process is very fast [4], but there also exist indirect processes in which absorbed photon energy is distributed among many degrees of freedom of the system [5]. Depending on the nature of the irradiated material, this may result in the emission (or desorption) of various charged and neutral species, from electrons to proteins [6], with a range of time delays; observation of the emission characteristics of such species can provide information about surface structure as well as the dynamics of surface processes.

In the case of silicon, which has been the subject of much research owing to its great importance to the electronics industry, it has been found that hydrogen surface atoms can be desorbed by direct single photon bondbreaking uv photodesorption [7] or resonant excitation of the $\mathrm{Si}-\mathrm{H}$ stretch mode [8], $\mathrm{Si}$ and $\mathrm{SiCl}$ can desorb when a $\mathrm{Si}$ surface melts under intense laser irradiation [9], and several species (e.g., $\mathrm{NO}$, atomic $\mathrm{Si}, \mathrm{Si}^{+}$ions, and $\mathrm{H}$ ) are thought to be desorbed when pairs of photogenerated holes break a Si surface bond [10].

In this Letter we report the photoemission of the exotic low mass atom positronium (Ps) [11] following positron implantation into a $p$-doped $\mathrm{Si}(100)$ surface. This effect, which is possible by virtue of the unique way in which Ps is produced on a Si surface [12], has a number of possible applications, such as studying surface electron dynamics in a manner analogous to regular photoemission [13], the production of short Ps (or positron) pulses, and very efficient Ps production in cryogenic environments. The last could be relevant to experiments in which antihydrogen is to be produced at low temperatures following the interaction of excited state Ps atoms with trapped antiprotons [14].

The experimental apparatus used for this work has been described in detail elsewhere [15] and so only a brief outline is given here. Positron plasmas generated with a two-stage Surko trap [16] are compressed in time to $\sim 1 \mathrm{~ns}$ (FWHM) using a high voltage electrostatic buncher [15] and then implanted into a Si target in a magnetic field of $\sim 0.16 \mathrm{~T}$. The resulting annihilation radiation is detected using a fast gamma ray detector, and single shot lifetime spectra are generated [17], from which the amount of Ps produced may be determined. We quantify this using the parameter $f_{d}$, defined as the fraction of lifetime spectra in the interval of 50 to $300 \mathrm{~ns}$ [18]. Because of the integration window used, the triplet to singlet 3:1 formation ratio, and lifetimes of $\sim 140$ and $0.125 \mathrm{~ns}$, respectively, we estimate that $f_{d} \sim 60 \%$ corresponds to a total positronium fraction (triplet plus singlet) of $100 \%$. The Si sample was cleaved from a $p-\operatorname{Si}(100)$ wafer $[2.7 \Omega \mathrm{cm}$ at $300 \mathrm{~K},(5.2 \pm 0.2) \times$ $10^{15}$ boron atoms $\mathrm{cm}^{-3}$ ] and etched in $\sim 2 \% \mathrm{HF}$, as described in Ref. [12]. Before our experiments were conducted the sample was heated to $\sim 950 \mathrm{~K}$ in the vacuum system to desorb hydrogen from the Si surface.

Figure 1 shows Ps lifetime spectra obtained with and without laser irradiation of a $p$-Si(100) target, demonstrating the Ps photoemission effect. When the laser was fired so as to overlap with the positron beam in time, an increase in $f_{d}$ was observed, indicating the emission of Ps associated with the light. The laser systems used here are described in Ref. [18]. Approximately 5 ns wide (FWHM) pulses of $532 \mathrm{~nm}$ light from a Nd:YAG laser were used to irradiate the Si sample, although any photon energy above the band gap $(1.13 \mathrm{eV})$ should produce similar effects. The laser spot size was $\sim 0.3 \mathrm{~cm}^{2}$. We estimate that the actual amount of energy absorbed by the Si will be $\sim 1 / 3$ of the total due to reflection at the tilted Si surface. 


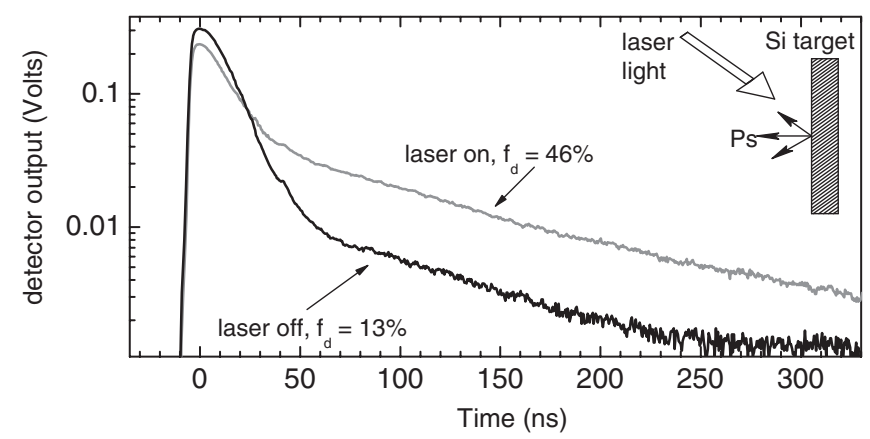

FIG. 1. Single shot lifetime spectra showing an increase in the amount of long-lived vacuum Ps due to the laser. The laser fluence was $6.4 \mathrm{~mJ} / \mathrm{cm}^{2}$. The inset shows the layout of the laser relative to the $\mathrm{Si}$ target and emitted Ps (with an exaggerated angle for clarity).

We find that the Ps yield depends on the laser fluence, as shown in Fig. 2(a). The delay time given in this figure refers to the difference between the positron and laser pulses, such that a negative delay means the laser is fired after the positrons. Figure 2(b) shows $f_{d}$ explicitly as a function of the absorbed laser fluence, $F$, for zero delay with respect to the positron implantation time. The Ps yield increases linearly at low values of $F$. This trend must

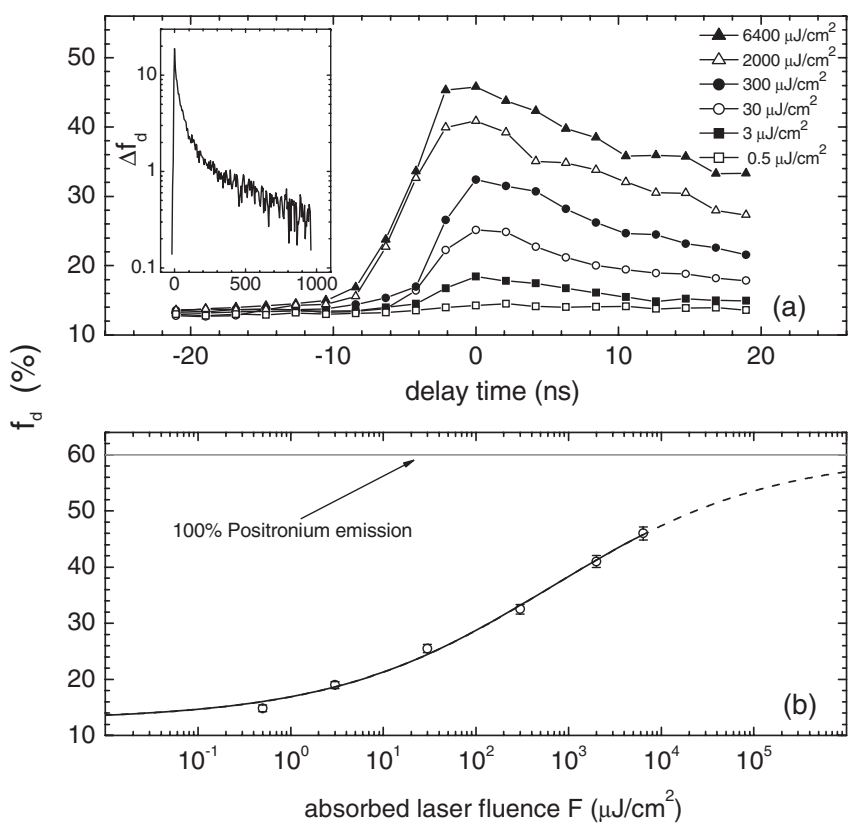

FIG. 2. Delayed fraction for different laser fluence and delay times (a) and versus laser fluence with zero delay (b). The sample temperature for these data is $300 \mathrm{~K}$. The inset in (a) shows the change in the delayed fraction caused by the laser over a longer time range for a $\sim 300 \mu \mathrm{J} / \mathrm{cm}^{2}$ pulse. The solid line in (b) is a fit using Eq. (1), extrapolated to higher laser fluence. A data point for $F=0$ is not shown in the figure but was included in the fit. The angle between the laser and the target was $\sim 10^{\circ}$. eventually saturate at $100 \%$ Ps formation (i.e., $f_{d} \sim$ $60 \%$ ), although other effects, such as melting [19], may limit $f_{d}$ to lower values.

In order to understand this behavior it is necessary to consider the mechanism by which Ps formation occurs on a Si surface [12]. This is related to a similar process wherein electrons excited to the conduction band by fs laser pulses may scatter (in a few hundred ps) into unoccupied surface states, and then form long-lived $(\tau \sim 80 \mathrm{~ns})$ surface excitons, $X[20]$. If a positron is present on the Si surface when this happens, there exists a positronic exciton level, Ps $X$ [12], and the electron may form Ps $X$ instead of $X$. In many respects the Ps $X$ and $X$ states are very similar, but with one important difference, namely, that $X$, involving a hole in the surface band, cannot leave the surface, whereas Ps $X$ is spontaneously emitted as a free Ps atom in vacuum. Our hypothesis that the $\operatorname{Ps} X$ and $X$ states are populated by similar mechanisms could in principle be tested by measuring the laser fluence dependence of the $X$ population in photoemission experiments [20] and comparing the results to those of Fig. 2(b).

The inset to Fig. 2(a) shows the long time dependence of the Ps photoemission yield. The general features of the decay are insensitive to the laser fluence used, and exhibit both a short and a long decay component. After $\sim 300 \mathrm{~ns}$ the Ps yield decays exponentially $(\tau \sim 0.7 \mu \mathrm{s})$. At this point, where $\Delta f_{d} \approx 1 \%$, the corresponding laser fluence would be about $0.3 \mu \mathrm{J} / \mathrm{cm}^{2}$, and the electron-hole $(e-h)$ density is $\sim 10^{16} \mathrm{~cm}^{-3}$ [21]. At this low density the decay is likely to be due to surface recombination, and the faster decay component at earlier times to Auger recombination [22] at a much higher $e$ - $h$ density.

The generation of $e-h$ pairs by the laser will result in a density $n_{e h}$ that increases at a rate proportional to the laser intensity, $\dot{n}_{e h} \propto I$ (where $I=F / \Delta t$, and $\Delta t$ is the laser pulse width). As our laser fluences are all $>0.3 \mu \mathrm{J} / \mathrm{cm}^{2}$, we are in the nonlinear regime, and the $e-h$ are destroyed via an Auger process [22] according to $\dot{n}_{e h}=-A n_{e h}^{2}$, giving at equilibrium a density $n_{e h} \propto \sqrt{F}$. Metastable surface electrons with areal density $n_{D}$ will accumulate in normally unfilled surface states [23] at a rate that depends on the $e-h$ density. The metastable surface electrons decay by filling holes in the $D_{\text {up }}$ band at a rate that depends on both the $e$ - $h$ density and on $n_{D}$. If we assume (a) that the density of surface holes that are able to accept a $D_{\text {down }}$ electron is proportional to $n_{e h}$ and (b) that excitation of already occupied $D_{\text {down }}$ electrons is needed to conserve energy and momentum, then the rate equation for $n_{D}$ is $\dot{n}_{D}=a_{1} n_{e h}^{\nu}-a_{2} n_{D}^{\mu} n_{e h}$, where $\nu$ and $\mu$ depend on the specific electronic recombination processes involved. In equilibrium, assumed to hold over the relatively long times of our laser pulses $(\sim 5 \mathrm{~ns})$, the density becomes $n_{D}=$ $a^{\prime} n_{e h}^{(\nu-1) / \mu}$. Then, if the Ps $X$ formation rate $R$ is proportional to $n_{D}$, we have $R(F) \propto F^{c}$, where $c=(\nu-1) / 2 \mu$. The Ps delayed fraction will then be 


$$
f_{d}(F)=f_{d}(0)+\left[f_{d}(\infty)-f_{d}(0)\right] R(F) /[R(F)+\gamma],
$$

where $\gamma$ is the decay rate of the surface positrons. Figure 2(b) shows a fit to our measurements using Eq. (1), from which we obtain $c=0.366 \pm 0.029$. This exponent is consistent with the growth and decay of the surface electron density $n_{D}$ both involving double Auger events, for which $\nu$ and $\mu$ would both equal 3, leading to $c=1 / 3$.

Figure 3 shows the Ps yield as a function of temperature with the laser fired at $t=0$ with different laser fluences. These data indicate that the Ps photoemission yield depends on both the laser fluence and sample temperature. The "laser off" data may be fitted by a thermally activated [24] Ps emission rate $R_{T}=S \exp \left\{-E_{a} / k T\right\}$, as has been previously observed [12]. When the laser is on, we add to this an $e-h$ plasma induced emission rate, $R_{L}=a+b T$, with a linear temperature dependence dictated by the observed linearity of $f_{d}(T)$ at the highest laser fluences of Fig. 3. The sum of these two rates is in competition with the surface annihilation rate $\gamma$ which results in a delayed fraction

$$
f_{d}(T)=f_{0}+f_{1}\left(R_{T}+R_{L}\right) /\left(\gamma+R_{T}+R_{L}\right) .
$$

Here $f_{0}$ is a background and $f_{1}+f_{0}=60 \%$ is taken to be the maximum delayed fraction (100\% Ps formation). The solid lines in Fig. 3 are fits of this function to the data. The reasonable quality of the fits supports the idea that the mechanism for laser-induced Ps photoemission includes a phonon-assisted component, as is implied by the form of $R_{L}$.

In our previous experiments with thermally generated Ps from the same sample [12], it was observed that the Ps is nearly monoenergetic, with a spread assumed to be due to scattering during the emission process. We confirm here that the photoemitted Ps has the same energy, as one would expect if it is produced via the same $\operatorname{Ps} X$ state. The Ps energy was determined by the Doppler shift of the $1 S-2 P$ transition line center, as described in Ref. [12]. Figure 4

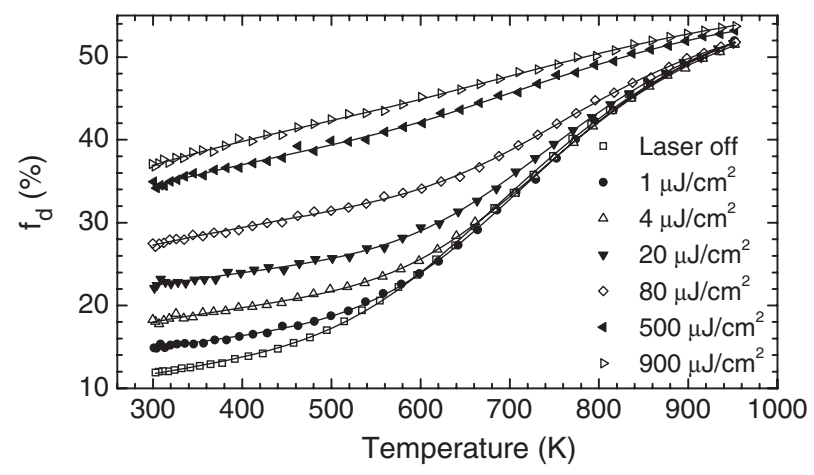

FIG. 3. Positronium yield as a function of temperature for various values of the absorbed laser fluence, as shown in the legend (with zero delay). The solid lines are fits to Eq. (2). shows line shapes measured with the lasers $(243 \mathrm{~nm}+$ $532 \mathrm{~nm}$ ) almost perpendicular to the sample surface $\left(\theta \sim 70^{\circ}\right)$. The double peaks arise due to the interaction of Ps with incident and reflected UV light. These data indicate that the mean longitudinal Ps energy is $0.155 \pm$ $0.007 \mathrm{eV}$, which is consistent with the $0.16 \pm 0.008 \mathrm{eV}$ observed for thermally produced Ps. The legend in Fig. 4 indicates the equivalent absorbed fluence of the $532 \mathrm{~nm}$ light that produces the same change in $f_{d}$ as was observed with the sample at $10^{\circ}$.

Extrapolation of the data of Fig. 3 suggests that one should be able to attain at least $50 \%$ Ps fractions in cryogenic environments, and probably more with higher laser fluence. In recent years, much attention has been directed towards the use of various porous [25] or channeled [26] insulating materials which efficiently produce Ps at low temperatures, and also provide a cooling mechanism [27-29]. Energetic $(\sim 1 \mathrm{eV})$ Ps is emitted from the bulk of these materials [30] and then cools via collisions with the internal surfaces, approaching the ambient sample temperature [29]. There is, however, a trade-off involved in this approach; Ps that has not thermalized will be emitted almost instantaneously, while colder Ps will be emitted more slowly, on a time scale commensurate with the Ps lifetime in the voids [18]. Thus, one may have a high yield of hot Ps that escapes into vacuum quickly, or a lower yield of colder Ps that takes some time to be emitted. Neither of these conditions are well suited to efficient laser excitation $[18,31]$ which requires short pulses of cold (or monoenergetic) atoms. This problem is avoided using materials from which Ps is formed via a surface state [32] (i.e., metals and semiconductors) since positron surface state lifetimes are typically short ( $<1 \mathrm{~ns})$. However, such Ps is usually emitted via a thermal desorption process [33], and hence cannot be used in cryogenic environments [34]. Given that Ps photoemission is very efficient, and that the Ps emission time is shorter than $\sim 0.7 \mathrm{~ns}$ (the positron surface state lifetime on $\mathrm{Si}$ ) [35], this new source of Ps is suitable for a wide range of experimental schemes (e.g., [14,36]).

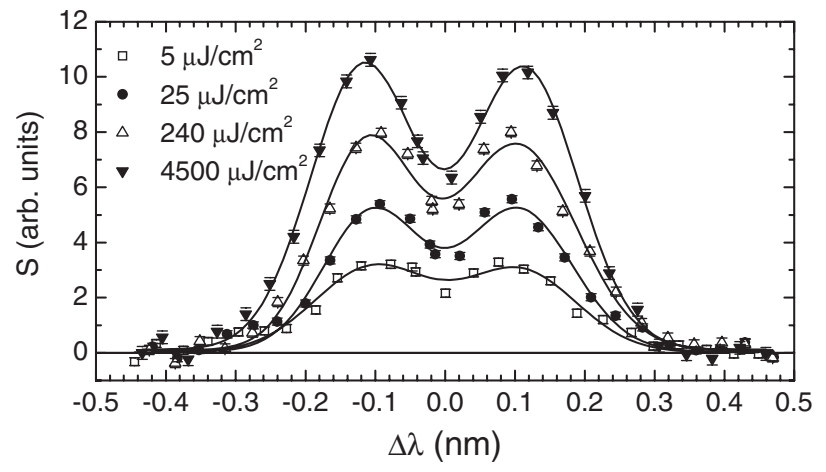

FIG. 4. Doppler shift measurements of Ps photoemitted from $\mathrm{Si}$ at $300 \mathrm{~K}$. The solid lines are fits of double Gauss functions. The wavelengths are given relative to the measured (unshifted) resonant value of $\lambda_{0}=243.02 \mathrm{~nm}$. 
It should be pointed out that Ps is also produced on $n$-type $\operatorname{Si}(100)$ via the same basic mechanism as for $p$-type. However, since the Fermi energy for $n$-type $\mathrm{Si}$ is above the $D_{\text {down }}$ level, these states are always filled and Ps is spontaneously produced without any laser irradiation. Preliminary measurements indicate that the spontaneous Ps yield from an $n$-type sample at room temperature is $\sim 50 \%$ of the maximum.

The production of extremely short pulses of Ps and of positrons may be possible using a Si based technique. As shown by Weinelt et al., the surface electron dynamics that populate the excitonic state occur on the time scale of $\sim 200$ ps (the time taken to populate the $D_{\text {down }}$ surface band) [20]. However, if an intense ps laser were used then this state will probably be populated via a much faster Auger process, as suggested above. Thus, if the laser pulse is applied after the positrons are implanted, Ps emission should occur on a time scale determined by the electronic population of the Ps $X$ state, since all of the available surface positrons will rapidly be removed as Ps atoms; this time could conceivably be of the order of a few tens of ps. Also, by varying the delay between the laser and positron pulses, the positron surface state lifetime could be directly measured. Moreover, if the subsequent Ps pulse were photoionized, one could generate similarly short positron pulses.

We have shown that $p$-Si(100) produces Ps with high efficiency when the sample is irradiated with laser light just prior to positron implantation. This occurs because Ps emitted from this material is formed via a positronic excitonlike state that may be populated by photogenerated electron-hole pairs, yielding nearly monoenergetic Ps. The photoemission mechanism should make it possible to efficiently produce short pulses of Ps in cryogenic environments.

This work was supported in part by the National Science Foundation under Grant No. PHY 0900919 and by the U.S. Air Force Research Laboratory.

[1] H. Hertz, Ann. Phys. (Berlin) 267, 983 (1887).

[2] A. Einstein, Ann. Phys. (Berlin) 322, 132 (1905).

[3] F. Reinert and S. Hüfner, New J. Phys. 7, 97 (2005).

[4] M. Schultze et al., Science 328, 1658 (2010).

[5] J. U. Andersen et al., J. Phys. B 35, R1 (2002).

[6] K. Tanaka et al., Rapid Commun. Mass Spectrom. 2, 151 (1988).

[7] T. Vondrak and X. Y. Zhu, J. Phys. Chem. B 103, 4892 (1999).

[8] Z. Liu et al., Science 312, 1024 (2006).
[9] J. Boulmer et al., J. Vac. Sci. Technol. A 9, 2923 (1991).

[10] J. Kanasaki et al., Phys. Rev. Lett. 82, 644 (1999).

[11] A. Rich, Rev. Mod. Phys. 53, 127 (1981).

[12] D. B. Cassidy et al., Phys. Rev. Lett. 106, 133401 (2011).

[13] M. Weinelt, J. Phys. Condens. Matter 14, R1099 (2002).

[14] A. Kellerbauer et al., Nucl. Instrum. Methods Phys. Res., Sect. B 266, 351 (2008).

[15] D. B. Cassidy, S. H. M. Deng, R. G. Greaves, and A.P. Mills, Jr., Rev. Sci. Instrum. 77, 073106 (2006).

[16] C. M. Surko and R. G. Greaves, Phys. Plasmas 11, 2333 (2004).

[17] D. B. Cassidy et al., Appl. Phys. Lett. 88, 194105 (2006).

[18] D. B. Cassidy et al., Phys. Rev. A 82, 052511 (2010).

[19] The Si surface will melt for $F \sim 1 \mathrm{~J} / \mathrm{cm}^{2}$. B. Strizker, A. Pospieszczyk, and J. Tagle, Phys. Rev. Lett. 47, 356 (1981).

[20] M. Weinelt et al., Phys. Rev. Lett. 92, 126801 (2004).

[21] Since the optical absorption coefficient of Si at $532 \mathrm{~nm}$ is $\alpha \sim 10^{4} \mathrm{~cm}^{-1}$ [e.g., M. A. Green and M. J. Keevers, Prog. Photovoltaics 3, 189 (1995)], and each photon produces 1 $e-h$ pair, the density neglecting recombination and diffusion is approximately $n_{e h}(F)=\alpha F / h \nu \approx 3 \times 10^{16} \times$ $F /\left(1 \mu \mathrm{J} / \mathrm{cm}^{2}\right)$.

[22] J. Dziewior and W. Schmid, Appl. Phys. Lett. 31, 346 (1977).

[23] These will be the $D_{\text {down }}$ dangling bond states for a clean surface. M.W. Weinelt et al., Appl. Phys. A 80, 995 (2005).

[24] The activation energy obtained for the present data was about twice the $0.25 \mathrm{eV}$ obtained in Ref. [12]. This could indicate that laser irradiation can affect the sample surface and change the way that the $\operatorname{Ps} X$ state is thermally populated.

[25] L. Liszkay et al., Appl. Phys. Lett. 92, 063114 (2008).

[26] S. Mariazzi et al., Phys. Rev. B 81, 235418 (2010).

[27] D. B. Cassidy et al., Phys. Rev. A 81, 012715 (2010).

[28] P. Crivelli et al., Phys. Rev. A 81, 052703 (2010).

[29] S. Mariazzi et al., Phys. Rev. Lett. 104, 243401 (2010).

[30] Ps is not bound in the bulk of metals or semiconductors due to screening by conduction electrons. J. Callaway, Phys. Rev. 116, 1140 (1959).

[31] F. Castelli et al., Phys. Rev. A 78, 052512 (2008).

[32] P. J. Schultz and K. G. Lynn, Rev. Mod. Phys. 60, 701 (1988).

[33] A. P. Mills, Jr., and L. Pfeiffer, Phys. Rev. Lett. 43, 1961 (1979).

[34] We note that oxygen on $\mathrm{Al}$ will produce Ps down to temperatures of $\sim 50 \mathrm{~K}$. [A.P. Mills, Jr., et al., Phys. Rev. Lett. 66, 735 (1991)]. However, these surfaces must be carefully prepared and require frequent cleaning.

[35] N. G. Fazleev et al., Phys. Rev. B 70, 165309 (2004).

[36] A. P. Mills, Jr., and M. Leventhal, Nucl. Instr. and Meth. B 192, 102 (2002). 